\title{
Atom-Molecule Laser Fed by Stimulated Three-Body Recombination
}

\author{
Bogdan Borca * Josh W. Dunn, Viatcheslav Kokoouline, and Chris H. Greene \\ JILA and Department of Physics, University of Colorado, Boulder, Colorado 80309-0440
}

(Dated: Submitted May 14, 2003)

\begin{abstract}
Using three-body recombination as the underlying process, we propose a method of coherently driving an atomic Bose-Einstein condensate (BEC) into a molecular BEC. Superradiant-like stimulation favors atom-to-molecule transitions when two atomic BECs collide at a resonant kinetic energy, the result being two molecular BEC clouds moving with well defined velocities. Potential applications include the construction of a molecule laser.
\end{abstract}

PACS numbers: 03.75.Mn,03.75.Pp,34.10.+x

One of the major goals of the recent research in BEC physics is the creation of a molecular condensate [1]. Many applications are envisioned for such a system, including coherent quantum chemistry [2]. One approach to forming a molecular BEC utilizes an atomic condensate along with a method of coherently driving pairs of atoms into (bound) molecular states. Two mechanisms have been proposed for this: (i) photoassociation, in which an external laser couples atomic states to molecular states [3], and (ii) control of the scattering length via external magnetic field ramps tuned near a Feshbach resonance [4]. Another problem of interest to the BEC community is the realization of an atom laser [5]. A proposed technique [6] for this is the application of a timedependent light pulse resulting in Bragg scattering of the condensate cloud, in which a fraction of the condensate receives a large momentum kick and attains velocity relative to the parent cloud.

In this Letter we propose a new mechanism for the formation of a molecular BEC from an atomic BEC. This mechanism relies on a twofold bosonic stimulation of the three-body recombination process - both by the number of atoms present and by the number of molecules. We show that two atomic condensates with a well-defined (resonant) relative velocity will mutually stimulate transitions into molecular states, resulting in the production of two coherent molecular clouds, each with a well-defined momentum.

Consider first the elementary process involving only three isolated atoms. The atoms, initially in an unbound state, are converted through recombination into a bound molecular state with $1 / 3$ of the recoil energy, while a third atom carries away the remaining $2 / 3$. Conservation of momentum requires that the resulting atom and molecule have momenta of equal magnitude $\left(\hbar k_{0}\right)$, but in opposite directions. The value of $k_{0}$ is fixed by conservation of energy to be $k_{0}=\sqrt{4 m E_{0} / 3 \hbar^{2}}$, where $E_{0}$ is the molecular binding energy and $m$ is the atomic mass. This process is characterized by a transition matrix element $T_{i f}$ between the initial and final three-body states. The spontaneous decay of an ultracold atomic sample of density $n$ through three-body recombination is usually characterized by a loss rate coefficient $K_{3}$, such that $d n / d t=-3 K_{3} n^{3} / 6$. Here $n^{3} / 6$ is the number of atomic triplets available for recombination in the considered volume and the factor of 3 denotes the atoms lost in each recombination. An additional factor of $1 / 6$ multiplies the right-hand side if all three atoms share the same state, i.e., if we consider the decay of a pure condensate [7]. The connection between $T_{i f}$ and $K_{3}$ is given by [8]

$K_{3}=\frac{6 / \hbar}{(2 \pi)^{2}} \int \frac{d^{3} \mathbf{k}}{d E}\left|T_{i f}\right|^{2} \delta\left(E-\frac{3 \hbar^{2} k_{0}^{2}}{4 m}\right) d E=\frac{6 k_{0} m}{\hbar^{3}}\left|T_{i f}\right|^{2}$.

If the recombination forms a weakly bound level with $E_{0} \approx \hbar^{2} /\left(m a^{2}\right)$ then $k_{0}=2 /(\sqrt{3} a)$, where $a$ is the $s$-wave scattering length. In this case, working in the ultracold limit and using momentum normalized states, Ref.[8] obtains $T_{i f}=\zeta \hbar^{2} a^{5 / 2} / m$ [8], where $\zeta$ is a numerical constant. More generally $K_{3}$ has been shown to scale with the fourth power of the scattering length in most cases, $K_{3}=\beta(\hbar / m) a^{4}[9,10]$ which for the weakly bound level gives $\beta=\left(12 \zeta^{2}\right) /(\pi \sqrt{3})$. Ref. [8] finds $\beta \approx 23$, while a more rigorous calculation, valid for a wide range of interatomic potentials [9], yields $\beta \approx 180$ for $a>0$ and $\beta \approx 1014$ for $a<0$. (We omit a $\sin ^{2}$ modulation factor discussed in $[9,10]$.)

We are interested in the process in which three-body recombination is stimulated by bosonic enhancement. We consider two atomic condensate clouds moving with relative velocity $\hbar k_{0} / m$, and choose a reference frame in which the two clouds move with equal velocities towards each other (see Fig. 1) along the direction $\hat{k}$. Let the wavevector of the atoms moving to the right (left) be denoted by $\mathbf{k}_{1}\left(\mathbf{k}_{2}\right)$ and the population of the mode by $n_{1}\left(n_{2}\right)$. Note that $\mathbf{k}_{1}=-\mathbf{k}_{2}=k_{0} / 2 \hat{k}$. Recombination of three atoms with momentum $\hbar \mathbf{k}_{1}$ can lead to a final state in which the resulting atom has momentum $\hbar \mathbf{k}_{2}$, in which case the resulting molecule has the momentum $\hbar \mathbf{k}_{\mathrm{m} 1}=2 \hbar k_{0} \hat{k}$. This transition will be strongly favored due to bosonic stimulation if the mode $\mathbf{k}_{2}$ is highly populated. We recall the fact that the magnitude of the momentum is fixed by energy and momentum conservation and only its direction has an arbitrary value. Therefore, it is possible that the atom-molecule pair is ejected along a direction different from $\hat{k}$. However, this process does not benefit from bosonic stimulation. By symmetry, re- 
combination of three atoms with momentum $\hbar \mathbf{k}_{2}$ will be strongly enhanced if the resulting atom-molecule pair is ejected along the direction $\hat{k}$. In this case the momentum of the molecule has the value $\hbar \mathbf{k}_{\mathrm{m} 2}=-2 \hbar k_{0} \hat{k}$ while the resulting atom has momentum $\hbar \mathbf{k}_{1}$. We denote the numbers of molecules with wavevectors $\mathbf{k}_{\mathrm{m} 1}$ and $\mathbf{k}_{\mathrm{m} 2}$ by $n_{\mathrm{m} 1}$ and $n_{\mathrm{m} 2}$, respectively. Finally, note that, for any threebody recombination the reverse process is also possible. For example, an atom with momentum $\hbar \mathbf{k}_{2}$ that collides with a molecule with momentum $\hbar \mathbf{k}_{\mathrm{m} 1}$ can induce it to break up, resulting in three atoms with momentum $\hbar \mathbf{k}_{1}$. In summary, the four modes considered here are coupled by the transitions

$$
\begin{aligned}
& 3 \mathrm{~A}\left(\mathbf{k}_{1}\right) \rightleftharpoons \mathrm{A}\left(\mathbf{k}_{2}\right)+\mathrm{A}_{2}\left(\mathbf{k}_{\mathrm{m} 1}\right), \\
& 3 \mathrm{~A}\left(\mathbf{k}_{2}\right) \rightleftharpoons \mathrm{A}\left(\mathbf{k}_{1}\right)+\mathrm{A}_{2}\left(\mathbf{k}_{\mathrm{m} 2}\right) .
\end{aligned}
$$

The many-body interaction Hamiltonian describing the reactions in Eqs. (10) is

$$
\hat{H}_{i}=\hbar \omega\left(c_{2}^{\dagger} c_{\mathrm{m} 1}^{\dagger} c_{1} c_{1} c_{1}+c_{1}^{\dagger} c_{\mathrm{m} 2}^{\dagger} c_{2} c_{2} c_{2}\right)+\text { h.c. }
$$

Here $c_{1,2}\left(c_{\mathrm{m} 1, \mathrm{~m} 2}\right)$ are the annihilation operators for the two atomic (molecular) modes and the coupling frequency is given by $\hbar \omega=T_{i f} / V^{3 / 2}$, where $V$ is the quantization volume. For a weakly bound molecule $\omega=\left[\zeta \hbar /\left(m a^{2}\right)\right]\left(a^{3} / V\right)^{3 / 2}$. The two operators,

$$
\begin{gathered}
\hat{N}=c_{1}^{\dagger} c_{1}+c_{2}^{\dagger} c_{2}+2 c_{\mathrm{m} 1}^{\dagger} c_{\mathrm{m} 1}+2 c_{\mathrm{m} 2}^{\dagger} c_{\mathrm{m} 2}, \\
\frac{\hat{P}}{\left(\hbar k_{0} / 2\right)}=c_{1}^{\dagger} c_{1}-c_{2}^{\dagger} c_{2}+4 c_{\mathrm{m} 1}^{\dagger} c_{\mathrm{m} 1}-4 c_{\mathrm{m} 2}^{\dagger} c_{\mathrm{m} 2},
\end{gathered}
$$

commute with the Hamiltonian in Eq. (2) as a consequence of the conservation of the total number of atoms and of the total momentum.

We now derive the equations of motion for the creation and annihilation operators in the Heisenberg representation. Adopting the notation $a_{1,2}(\tau)$ and $b_{1,2}(\tau)$ for the atomic and molecular Heisenberg operators divided by $\sqrt{N}$ (in which fast oscillations with the frequency associated to the non-interacting energy were factored out), the equations of motion read,

$$
\begin{aligned}
i \frac{d}{d \tau} a_{1} & =3 a_{1}^{\dagger} a_{1}^{\dagger} b_{1} a_{2}+b_{2}^{\dagger} a_{2} a_{2} a_{2}, \\
i \frac{d}{d \tau} a_{2} & =3 a_{2}^{\dagger} a_{2}^{\dagger} b_{2} a_{1}+b_{1}^{\dagger} a_{1} a_{1} a_{1}, \\
i \frac{d}{d \tau} b_{1} & =a_{2}^{\dagger} a_{1} a_{1} a_{1}, \\
i \frac{d}{d \tau} b_{2} & =a_{1}^{\dagger} a_{2} a_{2} a_{2} .
\end{aligned}
$$

We use a scaled time $\tau=\Omega t$ with $\Omega=N^{3 / 2} \omega=n^{3 / 2} T_{i f}$ ( $n=N / V$ is the total concentration of atoms). For a shallow molecular level $\Omega=\left[\zeta \hbar /\left(m a^{2}\right)\right]\left(n a^{3}\right)^{3 / 2}$. Equations (3)- (6) describe the dynamic population transfer

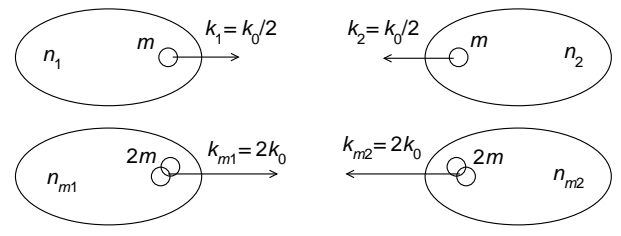

FIG. 1: Schematic diagram of the four possible states of the atoms: atomic states with momenta $\hbar \mathbf{k}_{1}$ and $\hbar \mathbf{k}_{2}$ have populations $n_{1}$ and $n_{2}$; molecular (molecular mass $2 m$ ) states with momenta $\hbar \mathbf{k}_{\mathrm{m} 1}$ and $\hbar \mathbf{k}_{\mathrm{m} 2}$ have populations $n_{\mathrm{m} 1}$ and $n_{\mathrm{m} 2}$.

between the atomic and molecular modes due to resonant three-body recombination. They are based on the assumption that all two-body elastic collisions (atomatom, molecule-molecule and atom-molecule) and also collisional loss of the weakly bound molecules to deeper molecular states can be neglected. In addition, we have neglected the spatial and temporal changes of the slowly varying envelopes of the condensate clouds. The simplest approximation to the above equation consists of replacing the operators by complex numbers equal to their averages: $\tilde{a}_{1,2}(t)=\left\langle a_{1,2}(t)\right\rangle$ and $\tilde{b}_{1,2}(t)=\left\langle b_{1,2}(t)\right\rangle$, i.e., the classical field approximation. The resulting system of ordinary differential equations, with initial conditions, can be integrated numerically. We consider the experimentally relevant case of an initial state with zero molecules. We also assume that initially the $N$ atoms are evenly split between the two momentum eigenstates: $N_{1,2} \equiv n_{1,2}(t=0)=N / 2$. Assuming real initial conditions, we set $\tilde{b}_{1,2}(0)=0$ and $\tilde{a}_{1,2}(0)=1 / \sqrt{2}$. The approximate version of the system of Eqs. (3)- (6) can be solved to describe the population transfer from the atomic to the molecular modes in a process characterized by the time constant $T_{R}=2 \pi / \Omega$. A characteristic that is similar to the superradiance phenomenon is double amplification (stimulation): after an initial buildup period for the molecular population, rates of further transitions are proportional both to the number of existing molecules in the recombined momentum state and to the number of atoms in their relevant momentum state.

The question of the validity of using the classical field approximation can be studied in general by accounting for higher-order terms in a cluster expansion of the fouroperator products in Eqs. (3)-(6) (see, e.g., Ref. [1]). However it is sufficient for our purpose to compare the results of the approximate version of Eqs. (3)- (6) to the many-body Schrödinger equation written using a basis of Fock states. We designate these basis states by $\left|n_{1}, n_{2}, n_{\mathrm{m} 1}, n_{\mathrm{m} 2}\right\rangle$ where $n_{1,2}\left(n_{\mathrm{m} 1, \mathrm{~m}, 2}\right)$ are the occupation numbers of the two atomic (molecular) modes. Due to the two conservation laws $n_{1}=N_{1}+n_{\mathrm{m} 2}-3 n_{\mathrm{m} 1}$ and $n_{2}=N_{2}+n_{\mathrm{m} 1}-3 n_{\mathrm{m} 2}$, we can restrict the basis set to only those accessible by three-body recombinations from our initial state and thus use only two occupation numbers, say $n_{\mathrm{m} 1}$ and $n_{\mathrm{m} 2}$, to parameterize the Fock states. 


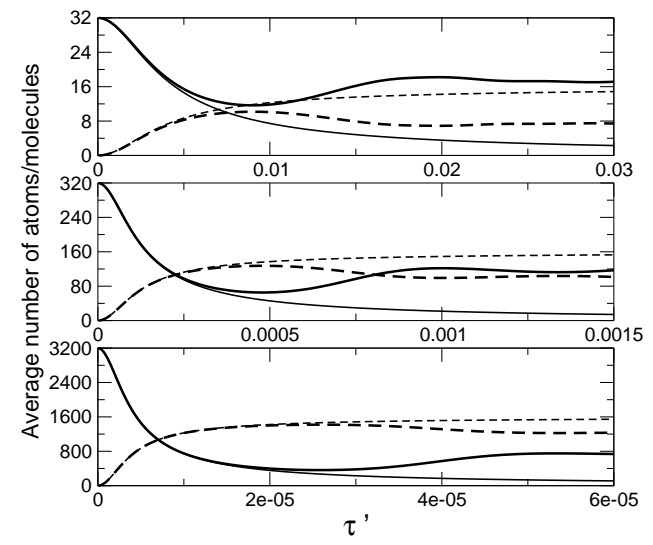

FIG. 2: Comparison of the scaled-time-dependent average number of atoms (solid lines) and molecules (dotted lines), obtained in two ways: by solution of the approximate Heisenberg equations (thick lines) and by solution of the many-body Schrödinger equation (thin lines). The three panels correspond to different total numbers of atoms $N$ : top $N=64$, middle $N=640$, bottom $N=6400$.

With this notation, the many-body wavefunction is

$$
|\Psi(t)\rangle=\sum_{n_{\mathrm{m} 1}, n_{\mathrm{m} 2}} f_{n_{\mathrm{m} 1}, n_{\mathrm{m} 2}}(t)\left|n_{\mathrm{m} 1}, n_{\mathrm{m} 2}\right\rangle .
$$

After eliminating the fast oscillations and introducing the time scaling $\tau^{\prime}=\omega t$, the Schrödinger equation becomes

$$
\begin{aligned}
& i \frac{d}{d \tau^{\prime}} f_{n_{\mathrm{m} 1}, n_{\mathrm{m} 2}} \\
& =\sqrt{\left(n_{1}+1\right)\left(n_{1}+2\right)\left(n_{1}+3\right) n_{2} n_{\mathrm{m} 1}} f_{n_{\mathrm{m} 1}-1, n_{\mathrm{m} 2}} \\
& +\sqrt{n_{1}\left(n_{1}-1\right)\left(n_{1}-2\right)\left(n_{2}+1\right)\left(n_{\mathrm{m} 1}+1\right)} f_{n_{\mathrm{m} 1}+1, n_{\mathrm{m} 2}} \\
& +\sqrt{\left(n_{2}+1\right)\left(n_{2}+2\right)\left(n_{2}+3\right) n_{1} n_{\mathrm{m} 2}} f_{n_{\mathrm{m} 1}, n_{\mathrm{m} 2}-1} \\
& +\sqrt{n_{2}\left(n_{2}-1\right)\left(n_{2}-2\right)\left(n_{1}+1\right)\left(n_{\mathrm{m} 2}+1\right)} f_{n_{\mathrm{m} 1}, n_{\mathrm{m} 2}+1} \text {. }
\end{aligned}
$$

Solving these equations with the initial conditions $f_{0,0}(0)=1$ and $f_{n_{\mathrm{m} 1}, n_{\mathrm{m} 2}}(0)=0$ for $n_{\mathrm{m} 1} \neq 0$ and $n_{\mathrm{m} 2} \neq 0$, allows us to calculate the average numbers of atoms populating any of the four considered modes. These averages are shown in Fig. 2 for different values of $N_{1}=N_{2}=N / 2$. Comparison with the corresponding results obtained using Eqs. (3)-(6) shows agreement over the initial period of fast transitions of atoms into molecular states. As expected, the classical field approximation fails at shorter times for lower values of $N$, while for sufficiently large $N$ it can be used to accurately describe the initial transition burst converting atoms to molecules. Equation (8) allows us to describe the population transfer within the four-mode approximation without making any additional approximations. However, calculations using the Fock basis become prohibitive if more than a few thousand atoms are considered.

Experimental arrangements that use the process described above to produce coherent molecular clouds can

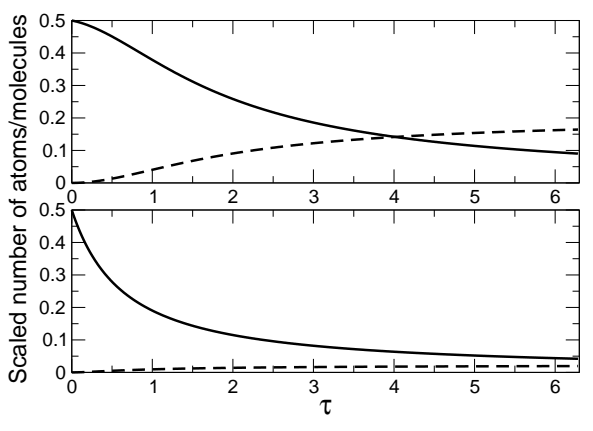

FIG. 3: Solutions of Heisenberg equations with elastic loss (top panel: $\gamma=0.1$, bottom panel $\gamma=1.5$ ). Solid line: atomic population fraction $\left|\tilde{a}_{1,2}(\tau)\right|^{2}$, dotted line: molecular population fraction $\left|\tilde{b}_{1,2}(\tau)\right|^{2}$.

be readily envisioned. The simplest experiment would begin with a time-dependent Bragg splitting of a single atomic cloud as in the experiment of Kozuma et al. [6] , but with the particular choice of momentum kick that matches the three-body resonance condition. In addition, the value of the molecular binding energy can be tuned using an external magnetic field [12, 13. In order to take full advantage of the stimulated transition into molecular states, (i.e., to transform a large fraction of the atomic clouds into molecular clouds) the collision time of the two atomic clouds should be comparable to the characteristic time of the three-body recombination process $t_{\text {coll }} \sim T_{R}$. Here $t_{\text {coll }} \approx R_{c} /\left(\hbar k_{0} / m\right)$ and $R_{c}$ is the characteristic size of the condensate along the collision direction. Consider a sample of ${ }^{85} \mathrm{Rb}$ with a concentration of $10^{15} / \mathrm{cm}^{3}$. Assuming that the scattering length is adjusted with the help of a Feshbach resonance to $a=400$ a.u. and that $E_{0}=\hbar^{2} / m a^{2}$, then we estimate $T_{R}=0.45 \mathrm{~ms}$ (using $K_{3}$ given by $[9]$ ). The resonant process described here is characterized by a finite width if additional processes that limit the lifetime of the involved states are considered. One such process is the collsionally induced decay of the molecular level to lower molecular states. Only a very rough estimation of the lifetime is available for molecules formed by atomic species commonly used in BEC experiments. Some estimations (see, e.g, [4]) cite values of at least $1 \mathrm{~ms}$ for this lifetime at molecular densities of $10^{15} / \mathrm{cm}^{3}$. In addition, recent experiments in ${ }^{85} \mathrm{Rb}$ [13] suggest that this is a conservative estimation, and that the actual lifetime might be somehow longer.

Up to this point we have neglected elastic collisions between atoms and molecules. In fact, if the resonant value of the relative velocity is not enforced by the experimental setup, depletion of the atomic clouds by elastic atomatom scattering is the main loss process taking place before the two condensate clouds separate [15]. As discussed in Ref. 15], elastic scattering must be accounted for beyond the mean-field (i.e., Gross-Pitaevskii) approximation in order to observe the condensate depletion due 
to elastic scattering. We can include this process in our simple model as a loss term in the interaction Hamiltonian. Considering only atom-atom collisions involving one atom with wavevector $\mathbf{k}_{1}$ and one with $\mathbf{k}_{2}$, Eqs. (3) and (4I) in the classical field approximation become

$$
\begin{aligned}
& i \frac{d}{d \tau} \tilde{a}_{1}=3 \tilde{a}_{1}^{*} \tilde{a}_{1}^{*} \tilde{b}_{1} \tilde{a}_{2}+\tilde{b}_{2}^{*} \tilde{a}_{2} \tilde{a}_{2} \tilde{a}_{2}-i \gamma\left|\tilde{a}_{2}\right|^{2} \tilde{a}_{1}, \\
& i \frac{d}{d \tau} \tilde{a}_{2}=3 \tilde{a}_{2}^{*} \tilde{a}_{2}^{*} \tilde{b}_{2} \tilde{a}_{1}+\tilde{b}_{1}^{*} \tilde{a}_{1} \tilde{a}_{1} \tilde{a}_{1}-i \gamma\left|\tilde{a}_{1}\right|^{2} \tilde{a}_{2} .
\end{aligned}
$$

Here the parameter $\gamma$ is the elastic scattering rate constant divided by $2 \hbar \Omega$, and is given by $\gamma=$ $\left(\hbar k_{0} / m\right) \sigma n /(2 \hbar \Omega)$ [15], where $\sigma$ is the elastic scattering cross section at the relevant two-body collision energy $E_{\text {coll }}=\hbar^{2} k_{0}^{2} / m$. Eqs. (9) show that the two processes resonant three-body recombination and condensate loss due to elastic scattering - compete with each other and that the value of the parameter $\gamma$ is crucial in determining which process dominates. Fig. 3 shows the solution of the Heisenberg equations that include the elastic loss terms for two extreme cases. The example with $\gamma=0.1$ illustrates the case of little elastic loss allowing almost $70 \%$ of the atoms to be transformed into molecules if $t_{\text {coll }} \approx T_{R}$. However, if $\gamma=1.5$, for the same collision time more than $80 \%$ of the atoms are lost due to elastic collisions and only about $8 \%$ end up in the molecular clouds.

The simplest estimation of $\gamma$ can be attempted in the low energy limit $\left(k_{0} \ll a^{-1}\right)$ where $\sigma \approx 8 \pi a^{2}$. This leads to $\gamma=(8 \pi / \sqrt{3} \zeta)\left(n a^{3}\right)^{-1 / 2}$ and yields high values of $\gamma$ even for very large values of the diluteness parameter (i.e., $n a^{3}=1$ corresponds to $\gamma=1.5$ ). However, note the importance of using an exact value of $\sigma$ corresponding to the correct $E_{\text {coll }}$. For example, in the case of a weakly bound molecular level $\left[E_{0} \approx \hbar^{2} /\left(m a^{2}\right)\right], k_{0} \approx 1 / a$, which is beyond the validity of the low energy approximation. Apparently, changing the value of the parameter $n a^{3}$ by changing either $n$ or $a$ is the primary way of shifting the balance between the two processes. However, special conditions might enhance or suppress either process. One such situation may occur when $a$ approaches zero and therefore elastic scattering vanishes while three-body recombination still has a considerable probability. Calculations by Esry et al. [9] predict a significant $K_{3}$ for $a=0$. Similarly, Stenger et al. 14 measured three-body recombination loss from $\mathrm{Na}$ condensates in the vicinity of Feshbach resonances, observing a large amount of loss even at small values of $a$. Consider the Feshbach resonance located near $B=535.7 \mathrm{G}$ in ${ }^{85} \mathrm{Rb}$. Tuning the magnetic field such that $a=0(B=537.6 \mathrm{G})$ leads to $E_{0}=75 \mu \mathrm{K}$, and for a density of $10^{16} / \mathrm{cm}^{3}$ we obtain $K_{3}=5.4 \times 10^{-32} \mathrm{~cm}^{6} / \mathrm{s}$ (as estimated in Ref. [9]), giving $T_{R} \approx 28 \mathrm{~ms}$. For Na with $a=0(B=907 \mathrm{G})$, Ref. 14. measures $K_{3} \approx 10^{-28} \mathrm{~cm}^{6} / \mathrm{s}$, giving $T_{R} \approx 0.24 \mathrm{~ms}$. Alternatively, one can choose the experimental conditions such that the elastic collisions are strongly suppressed exactly at the resonant value of the collision energy $E_{\text {coll }}$ due to the presence of a Ramsauer minimum (see, for example, 16]). The exact resonant matching between the position of the Ramsauer minimum and that of the binding energy of the molecular state (i.e., $E_{\text {coll }}=E_{0} / 3$ ) can be achieved near some Feshbach resonances. For the above mentioned resonance in ${ }^{85} \mathrm{Rb}$, this matching occurs for $B=547 \mathrm{G}$, when $a=-353$ a.u., and $E_{0}=1.05 \mathrm{mK}$. In this case, considering $n=10^{15} / \mathrm{cm}^{3}$, one finds $T_{R} \approx$ $1.3 \mathrm{~ms}\left(K_{3}=9.2 \times 10^{-26} \mathrm{~cm}^{6} / \mathrm{s} \mathrm{cf}\right.$. [9] $)$. Since producing an atomic condensate at negative $a$ might be a problem, we assume here that it is produced at some positive $a$ and that the magnetic field is swept into resonance precisely when the two atomic clouds start colliding.

In conclusion, resonant three-body recombination that benefits from the superradiant-like bosonic stimulation could be a promising candidate for producing coherent samples of molecules from existing atomic condensates. The simplest experimental setup that takes advantage of this process involves a collision of two atomic condensates, which produces two molecular condensates in counterpropagating momentum eigenstates. This setup could be particularly promising for the construction of a molecule laser by colliding two atomic condensates.

This work was supported in part by the NSF.

* On leave from the Institute for Space Sciences, Bucharest-Magurele 76900, Romania.

[1] P. Zoller, Nature (London), 417, 493 (2002).

[2] M. G. Moore and A. Vardi, Phys. Rev. Lett. 88, 160402 (2002).

[3] P. S. Julienne, K. Burnett, Y. B. Band, and W. C. Stwalley, Phys. Rev. A 58, R797 (1998).

[4] E. Timmermans et al., Phys. Rev. Lett. 83, 2691 (1999); E. Timmermans, P. Tommasini, M. Hussein, and A. Kerman, Phys. Rep. 315, 199 (1999).

[5] E. W. Hagley et al., Science 283, 1706 (1999).

[6] M. Kozuma et al., Phys. Rev. Lett. 82, 871 (1999).

[7] Y. Kagan et al., JETP Lett., 42, 209 (1985).

[8] P. O. Fedichev, M. W. Reynolds, and G. V. Shlyapnikov, Phys. Rev. Lett. 77, 2921 (1996).

[9] B. D. Esry, C. H. Greene, and J. P. Burke Jr., Phys. Rev. Lett. 83, 1751 (1999).

[10] E. Nielsen and J. H. Macek, Phys. Rev. Lett. 83, 1566 (1999); P. F. Bedaque, E. Braaten, and H. -W. Hammer, Phys. Rev. Lett. 85, 908 (2000).

[11] T. Köhler and K. Burnett, Phys. Rev. A 65, 033601 (2002).

[12] S. Inouye et al., Nature (London) 391, 151 (1998).

[13] E. A. Donley, N. R. Claussen, S. T. Thompson, C. E. Wieman, Nature (London) 417, 529 (2002).

[14] J. Stenger et al., Phys. Rev. Lett. 82, 2422 (1999).

[15] Y. B. Band, M. Trippenbach, J. P. Burke Jr., and P. S. Julienne, Phys. Rev. Lett. 84, 5462 (2000).

[16] J. P. Burke Jr., J. L. Bohn, B. D. Esry, and C. H. Greene, Phys. Rev. Lett. 80, 2097 (1998). 